Reveal ing nol ecul ar-I evel surface struct ure of anyl oi d fi brils in I i qui d by means of frequency modul at i on at om c force mi croscopy

\begin{tabular}{|l|l|}
\hline 著者 & $\begin{array}{l}\text { Fukuma Takeshi, Nost aert Ani ka S., Ser pel I } \\
\text { Loui se C., Jarvi s Suzanne P. }\end{array}$ \\
\hline 著者別表示 & 福間 岡I士 \\
\hline $\begin{array}{l}\text { j our nal or } \\
\text { publ i cat i on titl e }\end{array}$ & Nanot echnol ogy \\
\hline vol une & 19 \\
\hline number & 38 \\
\hline page range & 384010 \\
\hline year & 2008 09-24 \\
\hline URL & ht t p: //doi . or g/10. 24517/00039984 \\
\hline
\end{tabular}




\section{Revealing molecular-level surface structure of amyloid fibrils by frequency modulation atomic force microscopy in liquid}

Short title: Revealing molecular-level surface structure of amyloid fibrils

Takeshi Fukuma $^{1}$, Anika S. Mostaert ${ }^{2}$, Louise C. Serpell ${ }^{3}$ and Suzanne P. Jarvis ${ }^{2}$

1. Frontier Science Organization, Kanazawa University, Kakuma-machi, Kanazawa 920-1192, Japan

Tel.: +81-76-264-5638, Fax: +81-76-264-5636, E-mail: fukuma@staff.kanazawa-u.ac.jp

2. Conway Institute of Biomolecular and Biomedical Research, University College Dublin, Belfield,

Dublin 4, Ireland

Tel.: +353-1-716-6766, Fax: +353-1-716-6777, E-mail: Anika.Mostaert@ucd.ie

Tel.: +353-1-716-6780, Fax: +353-1-716-6777, E-mail: Suzi.Jarvis@ucd.ie

3. Department of Biochemistry, University of Sussex, John Maynard Building, Falmer BN1 9QG,

United Kingdom

Tel.: +44-1273-877-363, E-mail: L.C.Serpell@sussex.ac.uk

Corresponding author: Suzanne P. Jarvis Suzi.Jarvis@ucd.ie 


\section{Abstract}

We have investigated the surface structure of islet amyloid polypeptide (IAPP) fibrils and $\alpha$-synuclein protofibrils in liquid by frequency modulation atomic force microscopy (FM-AFM). Angstrom-resolution FM-AFM imaging of isolated macromolecules in liquid is demonstrated for the first time. Individual $\beta$-strands aligned perpendicular to the fibril axis with a spacing of $0.5 \mathrm{~nm}$ are resolved in FM-AFM images, which confirms cross- $\beta$ structure of IAPP fibrils in real-space. FM-AFM images also reveal the existence of $4 \mathrm{~nm}$ periodic domains along the axis of IAPP fibrils. Stripe features with $0.5 \mathrm{~nm}$ spacing are also found in images of $\alpha$-synuclein protofibrils. However, in contrast to IAPP fibrils, the stripes are oriented $30^{\circ}$ from the axis, suggesting the possibility of different $\beta$-strand alignment in protofibrils from that in mature fibrils or the regular arrangement of Thioflavin T molecules present during the fibril preparation aligned at the surface of the protofibrils.

PACS Code: 68.37.Ps, 81.07.-b, 87.14.em, 87.15.B- 


\section{Introduction}

Recent progress [1] in frequency modulation atomic force microscopy (FM-AFM) [2] has enabled true atomic resolution imaging in liquid with piconewton order loading forces [3]. This novel FM-AFM technique has several advantages over conventional atomic-scale imaging technique using contact-mode AFM [4]. Unlike contact-mode AFM, FM-AFM utilizes an oscillating stiff cantilever to probe the tip-sample interaction potential and hence is able to detect strong short-range interaction force without instabilities known as "jump-to-contact” [5]. This advantage has been demonstrated by imaging individual hydration layers formed on a lipid membrane, where the tip-sample distance control was made at each branch of the oscillatory force profile presented by the ordered water molecules [6]. In addition, FM-AFM can provide a better spatial resolution by reducing the cantilever oscillation amplitude and enhancing the sensitivity to the short-range interaction force [7]. This capability is also demonstrated by imaging lipid-ion networks formed at the water/lipid interface of a lipid membrane with sub-Angstrom resolution [8].

Although these striking results have highlighted the advantages of using FM-AFM over conventional AFM techniques, the capability of FM-AFM for imaging isolated biomolecules with Angstrom resolution remains untested. As the vertical motion of the cantilever in dynamic-mode AFM dramatically reduces the lateral friction force compared to that in contact-mode AFM, imaging of isolated biomolecules with Angstrom resolution should be possible. However, some requirements may 
hinder such applications, for example, the use of stiff cantilevers and self-excitation. Neither of these would directly result in high loading forces nor sample damage, yet they may impose more stringent requirements on tip-sample distance control (technical details will be discussed later). Thus, the applicability of FM-AFM to the imaging of relatively rough surfaces has often been questioned. In this study, we address these issues by imaging amyloid fibrils under solution conditions (biological macromolecular assemblies), deposited on a mica substrate.

Amyloid fibrils are formed from a variety of proteins which are normally soluble in physiological solution $[9,10]$. Under certain conditions, the precursor proteins misfold to form a protofibril having an elongated structure with a diameter of 3-5 nm and length of less than $100 \mathrm{~nm}$. The protofibrils grow to form fully matured amyloid fibrils which are typically $10-30 \mathrm{~nm}$ in diameter and up to several micrometers in length. The fully formed fibrils are insoluble $\beta$-sheet structures that can form aggregates in tissues and organs known as amyloid deposits. These deposits are found in a range of neurodegenerative diseases such as Alzheimer’s, Parkinson's and Huntington’s disease [9].

Understanding the structure of amyloid fibrils is essential for elucidating the nucleation and kinetics of fibrillation in relation to the pathogenic pathway of amyloidoses. While the structure of amyloid fibrils remains unclear, there are several defining features that are generally accepted. Amyloid fibrils commonly have a $\beta$-sheet-rich structure, which characteristically binds the histological dyes Thioflavin $\mathrm{T}$ and Congo red [11]. Their X-ray diffraction patterns show $\sim 0.5 \mathrm{~nm}$ along, and $\sim 1.0 \mathrm{~nm}$ reflections perpendicular to the fibril axis [12-14]. These distinctive X-ray diffraction patterns 
represent $\beta$-strands aligned perpendicular to the fibril axis and $\beta$-sheets aligned parallel to the axis consistent with a cross- $\beta$ arrangement. This arrangement has also been confirmed by cryo-electron microscopy images of Alzheimer's amyloid showing striations perpendicular to the fibre axis [15]. A detailed understanding of the generic and specific aspects of the structure of amyloid fibrils has yet to be conclusively determined $[16,17]$.

AFM has been used for studying the structure and formation of amyloid fibrils [18-21]. The highest resolution images have shown protofibril arrangement in a matured fibril with $\sim 10 \mathrm{~nm}$ resolution [19], and surface corrugations of polypeptide oligomers with $\sim 3 \mathrm{~nm}$ resolution [20]. Although this resolution is sufficient for visualizing the way in which protofibrils assemble into matured fibrils, molecular-scale surface structures (e.g., $\beta$-strand arrangements) have remained inaccessible. Angstrom-resolution imaging capability of FM-AFM offers novel AFM characterization of amyloid fibrils to provide a better understanding of their surface structure and mechanisms of assembly.

In this study, we have investigated the surface structure of amyloid fibrils formed from the islet amyloid polypeptide (IAPP) and $\alpha$-synuclein using an ultra low noise FM-AFM [22]. IAPP fibrils are composed of a 37-residue peptide and are associated with type 2 diabetes when deposited in pancreatic islets [23], whilst $\alpha$-synuclein fibrils are associated with Parkinson's disease. Molecular-level FM-AFM imaging of these fibrils is presented here, demonstrating the Angstrom-resolution imaging of isolated macromolecules in liquid for the first time. 


\section{Experimental}

Full-length IAPP (1-37) (Bachem) was dissolved in milli-Q water to a concentration of $20 \mathrm{mg} / \mathrm{ml}$ and incubated until required. Amyloid fibrils formed from IAPP (1-37) are known to spontaneously form at this high concentration [24]. The $\alpha$-synuclein fibril sample was prepared by shaking a $200 \mu \mathrm{l}$ solution containing $400 \mu \mathrm{M} \alpha$-synuclein, $300 \mathrm{mM}$ MOPS/KOH buffer (pH 7.2), $20 \mu \mathrm{M}$ Thioflavin T and $0.02 \%$ azide, at $37^{\circ} \mathrm{C}, 450 \mathrm{rpm}$ for 6 days and then leaving this solution for several days at room temperature without shaking [25]. For the IAPP sample, the stock solution was diluted to $10 \mu \mathrm{g} / \mathrm{ml}$ and $20 \mu \mathrm{l}$ was deposited onto freshly cleaved mica and left for $1 \mathrm{~h}$ without drying out. The sample was gently rinsed with milli-Q water, and $120 \mu \mathrm{l}$ phosphate buffered saline (PBS) solution was added immediately before FM-AFM imaging. In the case of $\alpha$-synuclein, $20 \mu \mathrm{l}$ of the stock solution was deposited directly onto freshly cleaved mica and left for $1 \mathrm{~h}$ without drying out. The sample was rinsed with milli-Q water, and $120 \mu \mathrm{l}$ milli-Q water was added prior to FM-AFM imaging.

A home-built FM-AFM with an ultra-low noise cantilever deflection detection system was used for imaging. The detailed design and performance of the system is described elsewhere [22]. Silicon cantilevers (NCH: Nanosensors) were used with spring constants of 20-40 N/m. The resonance frequency and Q factor in liquid were $120-150 \mathrm{kHz}$ and 8-12, respectively. The cantilevers were oscillated with a home-built self-excitation circuit with a constant amplitude feedback control, which adjusts the magnitude of the cantilever excitation signal to keep the oscillation amplitude constant. The frequency shift was detected with a commercially available phase-locked loop (PLL) circuit 
(EasyPLL: Nanosurf). The tip-sample distance regulation was made in constant frequency shift $(\Delta f)$ mode using a commercially available AFM controller (MFP-3D: Asylum Research). All experiments were performed in liquid (as described above) at room temperature.

\section{Results and Discussion}

\section{1. IAPP fibrils in PBS solution}

Figure 1(a) shows an FM-AFM image of IAPP fibrils deposited on mica obtained in PBS solution.

The image reveals a broad range of fibril length from 0.1 to $1 \mu \mathrm{m}$. Some fibrils appear to be bundles of protofilaments while others present a smoother surface. Figure 1(b) shows a cross-sectional plot

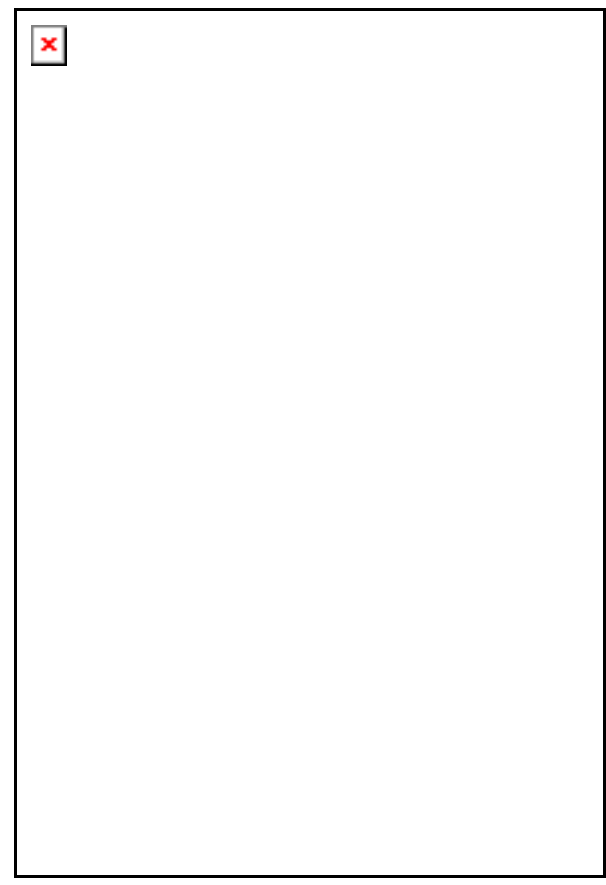

measured across two fibrils [line A-B in figure 1(a)]. The profile reveals that the distance between the adjacent fibrils is $\sim 15 \mathrm{~nm}$ and their height is $\sim 13 \mathrm{~nm}$. We measured the size of other fibrils in the same 
way and found that the diameter of the fibrils ranges from 7 to $13 \mathrm{~nm}$. This is in agreement with values of 5 to $15 \mathrm{~nm}$ reported previously [24].

With FM-AFM, the cantilever is oscillated with a self-excitation circuit, where the phase of the cantilever vibration is detected and used for producing a cantilever excitation signal. Thus, the cantilever oscillation can be disrupted by the occasional tip crash or adhesion, leading to a temporary loss of distance feedback control. Another potential problem is that FM-AFM requires a stiff $(>1$ $\mathrm{N} / \mathrm{m}$ ) cantilever for reducing the oscillation amplitude without instabilities and suppressing the cantilever thermal vibration. This will not cause problems if the distance feedback is functioning effectively. However, even a small error in the distance feedback regulation may result in a large loading force due to the high stiffness of the cantilever. Therefore, it has often been questioned if

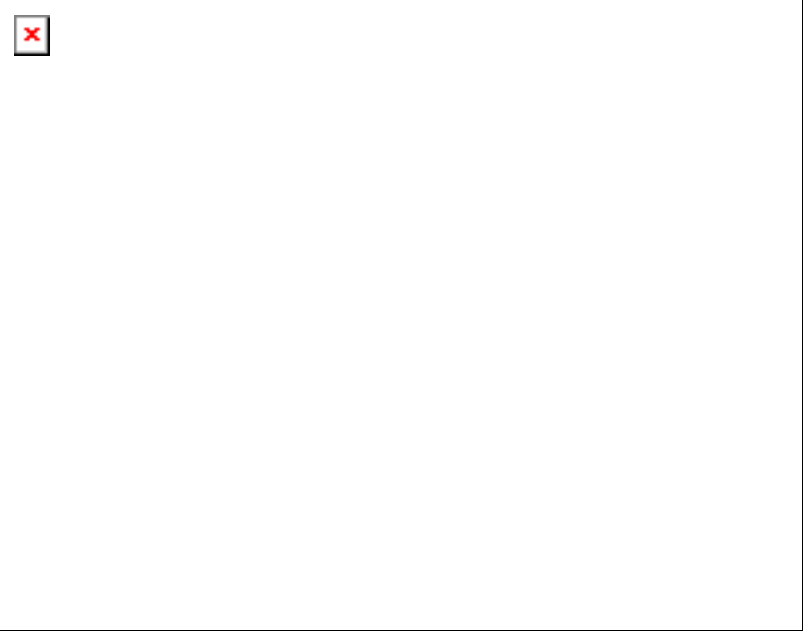


FM-AFM is capable of imaging an isolated large and fragile biological macromolecule without damage.

In this work, we attempt to resolve this question by imaging isolated amyloid fibrils with different imaging speed. We found that FM-AFM is capable of imaging the isolated fibrils ( 13 nm in height) without serious damage as long as the imaging speed was maintained below $1 \mu \mathrm{m} / \mathrm{sec}$ as shown in figure 1(a). Further increase of the imaging speed results in disruption of the cantilever oscillation or physical removal of the macromolecules from the surface.

Figure 2 shows an FM-AFM image taken at the center of the image shown in figure 1(a). The image shows that the surface of the fibril is not flat, but has a slight corrugation. This corrugation is more clearly seen in the cross-sectional plot [figure 2(b)] measured along the line A-B indicated in figure 2(a). The height of the corrugation is approximately $0.8 \mathrm{~nm}$. Although we could not find clear periodicity of the corrugation, the separation between the adjacent maxima was typically $\sim 40 \mathrm{~nm}$. IAPP fibrils formed from several coiling protofilaments show 25-50 nm crossover repeat, which is consistent with the periodicity of the observed height corrugation. Although the origin of the height corrugation is still unknown, it may be related to the formation of a bundle of twisted protofilaments.

In addition to the large corrugation, the cross-sectional plot in figure 2(b) reveals the existence of smaller periodic domains as indicated by the arrows. Note that the periodic domains are not parallel to the fast scan line, but perpendicular to the fibril axis. The same structure is also confirmed in the enlarged image of the same fibril as shown in figure 2(c). In order to show the contrast associated 
with the domains, the same image is shown with an enhanced contrast in figure 2(d). The cross-sectional plot in figure 2(e) reveals that the periodic domains have a corrugation height of $\sim 0.1$ $\mathrm{nm}$ and a periodicity of $\sim 4 \mathrm{~nm}$. The height corrugation is relatively small and the domains do not seem to have sharp boundaries. This suggests that the periodic structure may be due to a gradual change in the packing density or arrangement of $\beta$-strands with $4 \mathrm{~nm}$ periodicity.

Figure 3(a) shows an FM-AFM image of the same fibril as imaged in figure 2(c), but with a higher $x$ 
magnification. The image shows finely striped features perpendicular to the fibril axis. The distance between adjacent stripes is approximately $0.5 \mathrm{~nm}$ as indicated in figure 3(a). Figure 3(b) shows a magnified image taken at the position indicated by an arrow in figure 3(a). Although the image contrast is not very clear, the striped features with $0.5 \mathrm{~nm}$ spacing are also visible in the image. Figure 3(c) shows a cross-sectional plot measured along the line A-B indicated in figure 3(b). The profile also confirms the existence of $0.5 \mathrm{~nm}$ periodic structure superimposed on a larger scale corrugation (i.e., nanoscale corrugation shown in figure 2). These results show that the observed periodic structure is not associated with instrumentation noise, but represents real subnanometer structure. This demonstrates that FM-AFM is capable of imaging Angstrom-scale structures of isolated macromolecules in liquid.

Due to the curvature of the fibril surface, it is difficult to portray fine structure with high contrast in the image [figure 3(b)]. In addition, the nanoscale corrugation shown in figure 2 makes it difficult to identify the $0.5 \mathrm{~nm}$ periodicity even in the cross-sectional plot [figure 3(c)]. Although we are aware of the risk of introducing artifacts, we applied quadratic line-by-line subtraction process to the image shown in figure 3(b) as an attempt to subtract the background curvature. The high contrast image obtained [figure 3(d)], shows the stripe features running from top left to bottom right of the image. Figure 3(e) shows a cross-sectional plot measured along the line C-D indicated in figure 3(d). The lines A-B and C-D were measured at exactly the same position of the image. Comparing the profiles in figure 3(c) and 3(d), we found that the quadratic subtraction process reduces the influence from the 
nanometer-scale corrugation without giving a significant change in the corrugations corresponding to the fine stripes in this case.

Makin and Serpell [23] used X-ray and electron diffraction to reveal the existence of periodic structure along IAPP fibril axes with a characteristic spacing of $\sim 0.47 \mathrm{~nm}$. Since the periodicity corresponds to the hydrogen bonding spacing between the $\beta$-strands [figure 3(f)], the periodic structure was attributed to the $\beta$-strands aligned perpendicular to the fibril axis [24]. The fine stripes observed in this study (figure 3) have comparable $\sim 0.5 \mathrm{~nm}$ spacing aligned perpendicular to the fibril axis. Therefore, we attribute the striped features as the $\beta$-strands of IAPP fibrils. This demonstrates the possibility of FM-AFM to visualize individual $\beta$-strands in real space in a physiologically relevant liquid environment. This is significant progress beyond the resolution of images obtained with conventional AFMs ( 10 nm) [19].

\section{$3.2 \alpha$-synuclein protofibrils in water}

Elucidating the structure of protofibrils is of equal importance to that of mature fibrils, for example, when attempting to understand mechanisms of amyloid fibril assembly. In addition, evidence exists for $\alpha$-synuclein protofibrils (or oligomeric fibrillization intermediates) being pathogenic rather than the fully formed fibril itself $[26,27]$, further emphasizing the importance of understanding structural details throughout the process of fibrillization. 


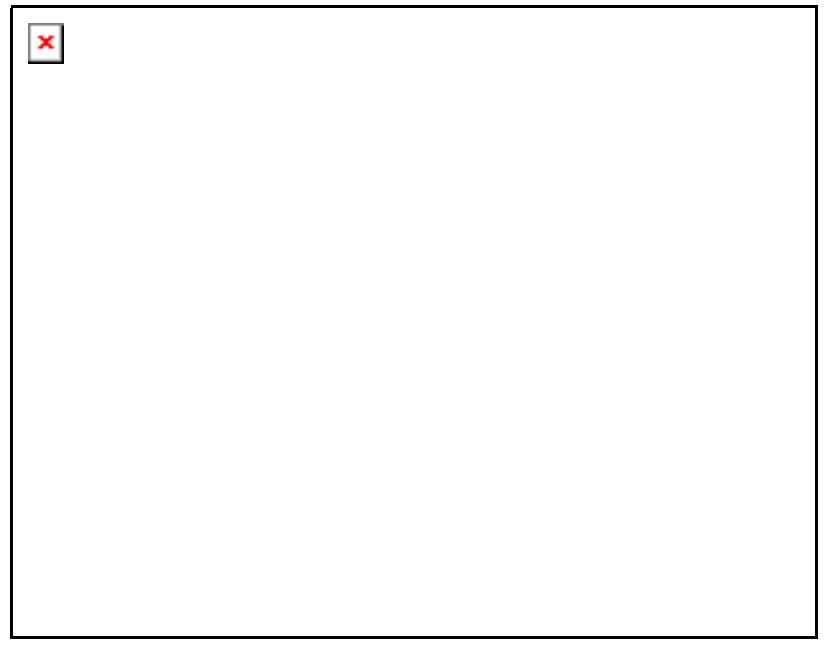

Figure 4: FM-AFM images of $\alpha$-synuclein protofibrils on mica in water. (a) $250 \mathrm{~nm} \times 250 \mathrm{~nm}$, tip velocity: $626 \mathrm{~nm} / \mathrm{sec}$, image height: $1.5 \mathrm{~nm} . \Delta f=+20 \mathrm{~Hz}$. (b) Cross-sectional plot measured along the line A-B indicated in (a). (c) $150 \mathrm{~nm} \times 150 \mathrm{~nm}$, tip velocity: $376 \mathrm{~nm} / \mathrm{sec}$, image height: $2.0 \mathrm{~nm} . \Delta f=$ $+20 \mathrm{~Hz}$. (d) Cross-sectional plot measured along the line C-D indicated in (c). The dotted lines in (a) and (c) show an averaging width of the profile.

Figure 4(a) shows an FM-AFM image of $\alpha$-synuclein protofibrils on mica in pure water. The image shows that the protofibrils are predominantly aligned forming an angle multiple of $60^{\circ}$. This arrangement of protofibrils reflects the six-fold symmetry of the mica substrate, suggesting a strong interaction between the mica substrate and the protofibrils. This interaction may account for the fact that we observe more protofibrils than matured fibrils although the stock solution should contain both.

The cross-sectional plot A-B [figure 4(b)] reveals that the height and width of the protofibrils are $\sim 1.2 \mathrm{~nm}$ and $\sim 8 \mathrm{~nm}$, respectively. Unlike the matured fibrils, the protofibrils do not have round-shaped cross-section. This asymmetrical cross-section may increase the area facing the mica surface and help to increase the interaction with the substrate.

An enlarged FM-AFM image shown in figure 4(c) reveals that the protofibrils consist of 2-3 protofilaments. A cross-sectional plot measured across the protofibril [figure 4(d)] shows that the 


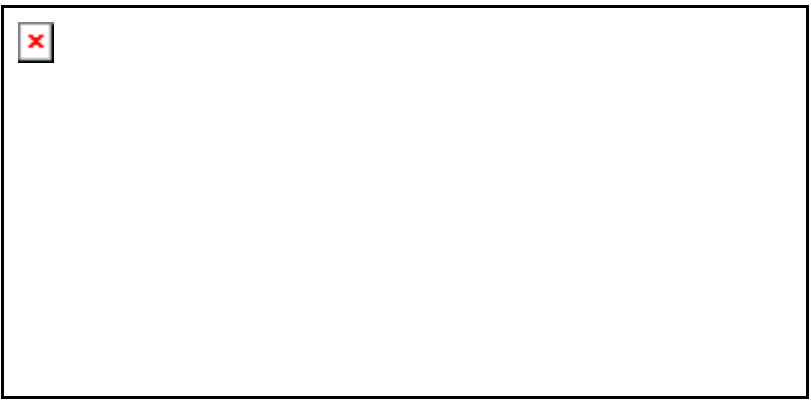

distance between the adjacent protofilaments is about 3-4 $\mathrm{nm}$. The protofilaments crossover at some locations along the protofibrils, but without showing clear periodicity. One such crossover is imaged in figure 5(a). The area indicated by the square in figure 5(a) is digitally-magnified to reveal the finely striped features as indicated by arrows in figure 5(b). The stripes have an approximate spacing of 0.5 $\mathrm{nm}$, which is expected for $\beta$-strands. However, the observed stripes are not oriented perpendicular to the protofibril axis, but instead at $30^{\circ}$ from the axis.

Whilst the observed stripes have dimensions indicative of individual $\beta$-strands, the angle does not match the cross- $\beta$ conformation reported for mature $\alpha$-synuclein fibrils [25]. It is possible that the protofibrils could acquire a different $\beta$-strand arrangement from that of matured fibrils. Another possibility is that the stripes are formed from Thioflavin $\mathrm{T}$ bound at regular intervals on an underlying $\beta$-strand arrangement which could indeed be aligned perpendicular to the fibril axis. Thioflavin T was present in the preparation of $\alpha$-synuclein but not IAPP fibrils. Confirmation for the reason the different alignment was observed in the case of $\alpha$-synuclein would require further experimental evidence. A possible future experiment would be to take force curves on the protofibrils while 
unraveling the individual stripes to see if there is a comparable mechanical response of the $\beta$-strands to that reported previously [21], and to observe the resulting effect on the images.

\section{Conclusions}

In this study, we have investigated the molecular level surface structure of mature IAPP fibrils and $\alpha$-synuclein protofibrils using ultra-low noise FM-AFM. We have demonstrated the Angstrom-resolution FM-AFM imaging of isolated macromolecules in liquid for the first time. The stable imaging of IAPP fibrils with a diameter of $~ 15 \mathrm{~nm}$ also revealed that FM-AFM is capable of imaging isolated macromolecules weakly bound to a substrate with a practical imaging speed (1 $\mu \mathrm{m} / \mathrm{sec})$.

FM-AFM images of IAPP fibrils showed the existence of $0.8 \mathrm{~nm}$ height corrugation with a period of $40 \mathrm{~nm}$ along the fibril axis, as well as smaller periodic domains with $0.1 \mathrm{~nm}$ height corrugations and $4 \mathrm{~nm}$ period along the fibril axis. In addition, individual $\beta$-strands aligned perpendicular to the fibril axis were resolved. These features confirmed the characteristic cross- $\beta$ structure of amyloid fibrils in real space.

FM-AFM images of $\alpha$-synuclein protofibrils revealed that they are composed of a bundle of 2-3 protofilaments, each having a diameter of 3-4 nm. The protofibrils showed striped structures with a spacing of approximately $0.5 \mathrm{~nm}$. However, the stripes are not aligned perpendicular to the fibril axis, but oriented $30^{\circ}$ away from the axis, suggesting the possibility of a different alignment of $\beta$-strands in 
the $\alpha$-synuclein protofibrils or the periodic binding of Thioflavin $\mathrm{T}$ at the surface of the protofibrils.

The capability of imaging individual $\beta$-strands with $0.5 \mathrm{~nm}$ resolution has illustrated a significant improvement from previous AFM resolution, and should be considered applicable to the study of other biomacromolecules. The technical features that brought this success include the small oscillation amplitude, high cantilever stiffness, and low deflection sensor noise [1, 22]. To date, X-ray and electron diffraction techniques have been intensively used for investigating the internal structure of biological molecules [17]. The results obtained in these experiments demonstrate that high-resolution FM-AFM has a unique capability of imaging surface structures of such biological macromolecules at Angstrom resolution in a physiologically-relevant environment. This capability should provide new insights into molecular mechanisms of a broad range of biological processes.

\section{Acknowledgements}

This research was funded by Science Foundation Ireland (grant no. 01/PI.2/C033). We are grateful to Shahin Zibaee, University of Cambridge, for preparing the $\alpha$-synuclein fibrils for these experiments. 


\section{References}

[1] Fukuma T, Kimura M, Kobayashi K, Matsushige K and Yamada H 2005 Rev. Sci.

Instrum. 76053704

[2] Albrecht T R, Grütter P, Horne D and Rugar D 1991 J. Appl. Phys. 69 668-73

[3] Fukuma T, Kobayashi K, Matsushige K and Yamada H, 2005 Appl. Phys. Lett. 87 034101.

[4] Ohnesorge F and Binnig G 1993 Science 260 1451-6

[5] Martin Y, Williams C C and Wickramasinghe H K 1987 J. Appl. Phys. 61 4723-9

[6] Fukuma T, Higgins M J and Jarvis S P 2007 Biophys. J. 92 3603-9

[7] Giessibl F J, Hembacher S, Bielefeldt H and Mannhart J 2000 Science 289 422-5

[8] Fukuma T, Higgins M J and Jarvis S P 2007 Phys. Rev. Lett. 98106101

[9] Westermark P, Benson M D, Buxbaum J N, Cohen A S, Frangione B, Ikeda S, Masters

C L, Merlini G, Saraiva M J and Sipe J D 2005 Amyloid 12 1-4

[10] Westermark P 2005 FEBS J. 272 5942-9

[11] Elghetany M T and Saleem A 1988 Stain Technol. 63 201-12

[12] Astbury W T, Dickinson S and Bailey K 1935 Biochem. J. 29 2351-60

[13] Eanes E D and Glenner G G 1968 J. Histochem Cytochem. 16 673-7

[14] Sunde M, Serpell L C, Bartlam M, Fraser P E, Pepys M B and F. Blake C C 1997 J. Mol. Biol. 273 729-39 
[15] Serpell L C and Smith J M 2000 J. Mol. Biol. 229 225-31

[16] Sawaya M R, Sambashivan S, Nelson R, Ivanova M I, Sievers S A, Apostol M I, Thompson M J, Balbirnie M, Wiltzius J J W, McFarlane H T, Madsen A Ø, Riekel C and Eisenberg D 2007 Nature 447 453-7

[17] Makin O S and Serpell L C 2005 FEBS J. 272 5950-61

[18] Jong K L D, Incledon B, Yip C M and DeFelippis M R 2006 Biophys. J. 91 1905-14

[19] Anderson M, Bocharova O V, Makarava N, Breydo L, Salnikov V V and Baskakov I V 2006 J. Mol. Biol. 358 580-96

[20] Mastrangelo I A, Ahmed M, Sato T, Liu W, Hough C W P and Smith S O2006 J. Mol. Biol. 358 106-19

[21] Fukuma T, Mostaert A S, Jarvis S P 2006 Tribology Lett. 22 233-7

[22] Fukuma T and Jarvis S P 2006 Rev. Sci. Instrum. 77043701

[23] Westermark P, Wernstedt C, Wilander E, Hayden D W, O’Brien T D and Johnson K H 1987 Proc. Natl. Acad. Sci. USA 84 3881-85

[24] Makin O S and Serpell L C 2004 J. Mol. Biol. 335 1279-88

[25] Serpell L C, Berriman J, Jakes R, Goedert M and Crowther R A 2000 Proc. Natl. Acad. Sci. USA 97 4897-902

[26] Goldberg M S and Landsbury Jr. P T 2000 Nature Cell Biol. 2 E115-9

[27] Lashuel H A, Hartley D, Petre B M, Walz T and Lansbury Jr. P T 2002 Nature 418 
